\title{
Estimation of dietary flavonoid intake and major food sources of Korean adults
}

\author{
Shinyoung Jun ${ }^{1}$, Sangah Shin ${ }^{2}$ and Hyojee Joung ${ }^{1,3 *}$ \\ ${ }^{1}$ Graduate School of Public Health, Seoul National University, 1 Gwanak-ro, Gwanak-gu, Seoul 08826, Korea \\ ${ }^{2}$ Graduate School of Medicine, The University of Tokyo, Tokyo 113-0033, Japan \\ ${ }^{3}$ Institute of Health and Environment, Seoul National University, Seoul 08826, Korea \\ (Submitted 11 March 2015 - Final revision received 13 August 2015 - Accepted 10 September 2015 - First published online 23 October 2015)
}

\section{Abstract}

Epidemiological studies have suggested that flavonoids exhibit preventive effects on degenerative diseases. However, lack of sufficient data on flavonoid intake has limited evaluating the proposed effects in populations. Therefore, we aimed to estimate the total and individual flavonoid intakes among Korean adults and determine the major dietary sources of these flavonoids. We constructed a flavonoid database of common Korean foods, based on the food list reported in the 24-h recall of the Korea National Health and Nutrition Examination Survey (KNHANES) 2007-2012, using data from the Korea Functional Food Composition Table, US Department of Agriculture flavonoid database, Phenol-Explorer database and other analytical studies. This database, which covers $49 \%$ of food items and $76 \%$ of food intake, was linked with the 24 -h recall data of 33581 subjects aged $\geq 19$ years in the KNHANES 2007-2012. The mean daily intake of total flavonoids in Korean adults was $318.0 \mathrm{mg} / \mathrm{d}$, from proanthocyanidins (22.3\%), flavonols (20.3\%), isoflavones (18.1\%), flavan-3-ols (16.2\%), anthocyanidins $(11.6 \%)$, flavanones $(11.3 \%)$ and flavones $(0.3 \%)$. The major contributing food groups to the flavonoid intake were fruits (54.4\%), vegetables (20.5\%), legumes and legume products $(16 \cdot 2 \%)$ and beverages and alcohols (3.1\%), and the major contributing food items were apples $(21.9 \%)$, mandarins $(12.5 \%)$, tofu (11.5\%), onions (9.6\%) and grapes (9.0\%). In the regression analysis, the consumption of legumes and legume products, vegetables and fruits predicted total flavonoid intake the most. The findings of this study could facilitate further investigation on the health benefits of flavonoids and provide the basic information for establishing recommended flavonoid intakes for Koreans.

Key words: Flavonoid database: Common Korean foods: Flavonoid intake: Flavonoid dietary sources: Korea National Health and Nutrition Examination Survey

Phytochemicals are synthesised by plants and are well-known to exhibit health protective properties. A major group of phytochemicals is polyphenols, largely comprised of flavonoids. The basic structure of flavonoids is a benzo- $\gamma$-pyrone ring, a functional hydroxyl group that performs antioxidant activities by scavenging free radicals and chelating mineral ions ${ }^{(1-3)}$. Dietary flavonoids are an integral part of the human diet and have received much attention in recent years as non-essential nutrients. Based on their chemical structure, dietary flavonoids are commonly categorised into six principal subclasses: flavonols, flavones, flavanones, flavan-3-ols and their oligomers and polymers, anthocyanidins and isoflavones. The oligomers and polymers of flavan-3-ols are also labelled as proanthocyanidins as a distinct subclass ${ }^{(4)}$. The antioxidant properties and related health effects differ among these subclasses ${ }^{(5,6)}$.

Many epidemiological studies have shown that dietary flavonoids are associated with lower incidences of degenerative diseases such as $\mathrm{CVD}^{(7-9)}$, type 2 diabetes $^{(10,11)}$, dementia ${ }^{(12,13)}$ and cancer ${ }^{(14-16)}$ - for example, flavonols showed a protective effect against type 2 diabetes in a Framingham Offspring cohort study $^{(17)}$, and anthocyanidins, flavan-3-ols, flavones and flavonols were individually associated with lower mortality caused by CVD in the Cancer Prevention Study II Nutrition Cohort $^{(7)}$. However, many knowledge gaps still exist in this field. Some studies have reported inconsistent associations ${ }^{(18-22)}$, and the underlying mechanisms have not been fully clarified $^{(23)}$. Thus, further studies, especially large and long-term trials, are needed to develop dietary recommendations ${ }^{(24,25)}$. One of the critical difficulties in such research lies in the ability to accurately estimate flavonoid intake.

Assessing dietary flavonoid intakes and their major food sources is the first step in documenting associations between flavonoids and diseases. Nonetheless, the lack of data about the flavonoid content of foods has limited our ability to estimate the flavonoid intakes among populations. The major challenges in estimating dietary flavonoid intakes are associated with the high variability in the flavonoid content of foods across different locations and types of cultivation, even within the same crop

Abbreviations: KFDB, flavonoid database of common Korean foods; KNHANES, Korea National Health and Nutrition Examination Survey.

* Corresponding author: H. Joung, fax +82 2883 2832, email hjjoung@snu.ac.kr 
variety, especially given the large number of individual flavonoid components ${ }^{(26,27)}$. To date, several countries have established national databases on flavonoids, including the $\mathrm{USA}^{(28-30)}$ and France ${ }^{(31-33)}$. In addition, a small number of studies on the estimation of flavonoid intakes and their major dietary sources has been published, but most of these studies have targeted populations other than Korean ${ }^{(34-36)}$. A few studies that have focused on the flavonoid intake of Koreans have reported intakes only from fruits and vegetables ${ }^{(37,38)}$ or are based on foreign databases ${ }^{(39)}$.

The Korea National Academy of Agricultural Science recently released the Korea Functional Food Composition Table, incorporating the concentrations of flavonoids and other compounds ${ }^{(40)}$. Although the table includes the flavonoid contents of selected foods cultivated in Korea, it still requires further development to assess common Korean diet. We tried to expand the table to construct a new flavonoid database of common Korean foods (KFDB) to cover the food items typically consumed by the Korean population. To the best of our knowledge, this is the first study to assess the flavonoid intake of the Korean population using a flavonoid database including seven flavonoid subclasses (the above-mentioned six subclasses and proanthocyanidins) and covering not only plant foods but also other food groups such as legumes and beverages and alcohols. We aimed to estimate the total and individual flavonoid intakes among Korean adults and determine the major dietary sources of these flavonoids.

\section{Methods}

\section{Study population}

The Korea National Health and Nutrition Examination Survey (KNHANES), conducted by the Korea Centers for Disease Control and Prevention (KCDC), is a national surveillance system that assesses the health and nutritional status of Koreans. To date, the survey has been executed in five rounds (1998, 2001, 2005, 2007-2009 and 2010-2012). The fourth and fifth rounds were conducted annually, throughout the year to avoid seasonal bias in diet. The subjects of each round were selected to represent the Korean population, aged 1 year and older, using a multistage clustered probability design. Written informed consent was obtained from all the subjects.

Among 45044 subjects who had completed a 24-h recall in the fourth and fifth KNHANES, 33581 subjects, aged 19 years and older, were selected for this study. The KNHANES data collection was approved by the KCDC Institutional Review Board, and this study was approved by the Institutional Review Board of the Seoul National University.

\section{Collection of diet and lifestyle information}

Trained dietitians conducted a nutrition survey at each participant's home to obtain information on dietary intake and dietary behaviour. Dietary intake data collected through a 1-d 24-h recall were transformed into individual food intakes using the KNHANES recipe database. Dietary behaviour data collected through questionnaires comprised the following variables. With respect to supplement use, the participants were asked whether they had taken supplements or not in the fourth KNHANES and whether they had taken supplements more than once a week during the month before the interview in the fifth KNHANES. Breakfast consumption was divided into two categories: 'yes' (ate breakfast on both the days before the interview) or 'no'. Food security was divided into four categories: full food security (able to eat an adequate amount and variety of food), marginal food security (able to eat an adequate amount but not variety of food), low food security (sometimes unable to afford enough food) and very low food security (often unable to afford enough food).

A structured questionnaire including information on sociodemographic and lifestyle factors was administered by trained interviewers at mobile examination centres. Household income was divided into four categories: low income (first quartile), middle-low income (second quartile), middle-high income (third quartile) and high income (fourth quartile). Educational level was divided into four categories: elementary school and lower, middle school, high school and college and higher. Regular alcohol consumption was divided into two categories: 'yes' (drank more than once a month during the year before the interview) or 'no'. Current smoking was divided into two categories: 'yes' (smoked $>100$ cigarettes in their lifetime and are still smoking) or 'no'. Physical activity was divided into two categories: 'active' (performed vigorous physical activity that requires a large amount of effort and causes rapid breathing e.g. running, fast cycling and playing football for more than $20 \mathrm{~min}$ - on more than $3 \mathrm{~d}$ during the week before the interview) or 'inactive'.

\section{Construction of the flavonoid database and estimation of flavonoid intake}

We constructed a KFDB. The food list in the database is comprised of 3193 foods consumed by the subjects of the fourth and fifth KNHANES. The KFDB was constructed based on the Korea Functional Food Composition Table ${ }^{(40)}$, US Department of Agriculture (USDA) flavonoid database for selected foods ${ }^{(28-30)}$ and the Phenol-Explorer database $e^{(31-33)}$. For the food items without flavonoid content in these databases, values from published articles were used after quality assessment according to the modified USDA data quality evaluation system ${ }^{(41)}$. The values for remaining food items were replaced with estimated values from similar food items, using the moisture conversion factors and logical zeros. Moisture conversion factors developed by the KCDC were applied for food items that have a similar nutrient composition but different processing or preparation methods, based on the criteria developed by Chun et $a l{ }^{(35)}$. Logical zeros were applied when the food groups were expected to contain no flavonoids, such as meats and poultry and fish and shellfish. The database included seven major flavonoid subclasses and their thirty-one main flavonoid aglycones: flavonols (quercetin, kaempferol, myricetin and isorhamnetin), flavones (luteolin and apigenin), flavanones (eriodictyol, hesperetin and naringenin), flavan-3-ols (catechin, epicatechin, epigallocatechin, theaflavin, theaflavin-3-gallate, theaflavin $3^{\prime}$-gallate, theaflavin 3,3'-digallate and thearubigin), 
anthocyanidins (cyanidin, delphinidin, malvidin, pelargonidin, peonidin and petunidin), isoflavones (daidzein, genistein and glycetin) and proanthocyanidins (dimers, trimers, 4-6 monomers, 7-10 monomers and >10 monomers).

We linked food consumption data from the KNHANES with the developed KFDB to estimate the subjects' flavonoid intake. Flavonoid content was expressed as aglycones $(\mathrm{mg} / \mathrm{d})$. The individual flavonoid intake from a food item was calculated by multiplying the flavonoid content by the total grams of food intake. The daily individual flavonoid intake was the sum of each individual flavonoid intake from all the food sources reported in the 1-d 24-h recall data. The daily total flavonoid intake was determined by the summation of the daily individual flavonoid intakes.

\section{Dietary sources of flavonoid intake}

We determined the major dietary sources of flavonoid intake. To identify the major food group sources of the total and subclasses of flavonoid intake, eighteen food groups were categorised based on the food group criteria of the KNHANES: grains, potatoes and starches, sugars and sweets, legumes and legume products, nuts and seeds, vegetables, mushrooms, fruits, meats and poultry, eggs, fish and shellfish, seaweeds, milk and dairy products, oils and fats, beverages and alcohols, seasonings, prepared foods and others. Furthermore, to estimate the predictive effect of the specific food groups including legumes and legume products, vegetables, fruits and beverage and alcohols, serving size $(\mathrm{g}$ ) for each food item was assigned based on the Korean Food Guidance System as described by Jung et $a l .^{(42)}$. The daily servings of a food item consumed by each subject were calculated by dividing the intake of the food by one serving size of the food item.

\section{Statistical analysis}

All the statistical analyses were carried out using SAS software (version 9.3; SAS Institute). Clusters, strata and survey weights were applied to all the analyses to adjust for the complex survey design of the KNHANES, which enabled the results to represent the Korean population. The daily intakes of flavonoids of the subpopulations grouped by socio-demographic and lifestyle factors were described using the means with their standard errors. The differences among flavonoid intakes of the subgroups after adjusting for the total energy intake were determined by Student's $t$ test and ANOVA using the SURVEYREG procedure. The contribution of each individual flavonoid intake to the intake of total flavonoids and subclasses was calculated as a percentage. The contribution of each food and food group to the intake of total flavonoids and subclasses was also calculated as a percentage. We also carried out multiple regression analysis to determine the extent to which total flavonoid and subclass intakes were explained by the intake of specific food groups. The model included the daily consumption of servings of legumes and legume products, vegetables, fruits and beverages and alcohols as independent variables. Factors that showed a significant difference in the $t$ test and ANOVA were included in the model for statistical control: survey phase, sex, household income, education level, regular alcohol drinking, current smoking, supplement use, breakfast consumption, food security and total energy intake. These variables showed no substantial collinearity or skewness. Statistical significance was accepted at $P<0.05$, and all the $P$ values were two-sided.

\section{Results}

\section{Evaluation of the developed flavonoid database for common Korean foods}

The developed KFDB covered 49\% (1579 items of 3193 items) of foods reported in the KNHANES 2007-2012 24-h recall data. The coverage was high in fruits (90\%), seaweeds (85\%), legumes and legume products (79\%), potatoes and starches (73\%), nuts and seeds (72\%), prepared foods (62\%) and vegetables (54\%), but low in oils and fats (33\%), mushrooms (32\%), milk and dairy products (30\%), beverages and alcohols (23\%), seasonings $(23 \%)$, grains $(22 \%)$, sugars and sweets (19\%) and others (14\%). The coverage for meats and poultry and fish and shellfish was $100 \%$ due to the application of logical zero. As a result, $76 \%$ of the total amount of food consumed by all the participants was covered by the KFDB.

\section{Estimated daily flavonoid intake}

The daily flavonoid intakes of subjects are shown in Table 1. The mean total flavonoid intake among the subjects was $318 \cdot 0$ (SE $4 \cdot 1) \mathrm{mg} / \mathrm{d}$. When the total energy intake was adjusted, total flavonoid intake was higher in women $(P<0 \cdot 001)$, non-regular alcohol consumers $(P<0.001)$, non-current smokers $(P<0.001)$, supplement users $(P<0.001)$ and breakfast eaters $(P<0.001)$ compared with their counterparts. Energy-adjusted total flavonoid intake increased with household income $(P<0.001)$, education level $(P<0.001)$, eating out frequency $(P<0.001)$ and food security $(P<0.001)$. The $50-64$ years age group had the highest intake of total flavonoids after energy adjustment, followed by the $30-49,65-74, \geqq 75$ and $19-29$ years age groups. With respect to the flavonoid subclasses, proanthocyanidins were the major contributors $(22 \cdot 3 \%)$ to the total flavonoid intake, followed by flavonols $(20 \cdot 3 \%)$, isoflavones (18.1\%), flavan-3-ols (16.2\%), anthocyanidins (11.6\%) and flavanones $(11.3 \%)$; flavones $(0.4 \%)$ accounted for the lowest proportion. The contributions of individual flavonoids to the intake of total flavonoid and subclasses are shown in Table 2.

\section{Major dietary sources of flavonoid intake}

The main food group contributors to flavonoid intake are shown in Table 3. The major dietary food group sources of total flavonoids were fruits $(54.4 \%)$, followed by vegetables (20.5\%), legumes and legume products (16.2\%), grains (4.5\%) and beverages and alcohols $(3 \cdot 1 \%)$. The main contributors to the intake of flavonols, flavones and anthocyanidins were vegetables and fruits. Fruits were the most important contributors to the intakes of flavanones and flavonols, and legumes and legume products were the most important 
Table 1. Daily dietary flavonoid intakes of Korean adults by socio-demographic and lifestyle factors (Mean values with their standard errors)

\begin{tabular}{|c|c|c|c|c|c|c|c|c|c|c|c|c|c|c|c|c|c|}
\hline \multirow[b]{3}{*}{ Subgroups } & \multirow{3}{*}{$\begin{array}{c}\text { Stratified } \\
\text { sample }(n)\end{array}$} & \multicolumn{16}{|c|}{ Flavonoid intake $(\mathrm{mg} / \mathrm{d})$} \\
\hline & & \multicolumn{2}{|c|}{ Flavonols } & \multicolumn{2}{|c|}{ Flavones } & \multicolumn{2}{|c|}{ Flavanones } & \multicolumn{2}{|c|}{$\begin{array}{l}\text { Flavan- } \\
\text { 3-ols }\end{array}$} & \multicolumn{2}{|c|}{ Anthocyanidins } & \multicolumn{2}{|c|}{ Isoflavones } & \multicolumn{2}{|c|}{ Proanthocyanidins } & \multicolumn{2}{|c|}{ Total flavonoids } \\
\hline & & Mean & SE & Mean & SE & Mean & SE & Mean & SE & Mean & SE & Mean & SE & Mean & SE & Mean & SE \\
\hline Participants & 33581 & 64.6 & 0.8 & $1 \cdot 0$ & 0.1 & 35.9 & $2 \cdot 2$ & $51 \cdot 4$ & $1 \cdot 7$ & $37 \cdot 0$ & $1 \cdot 3$ & $57 \cdot 5$ & 0.9 & $70 \cdot 8$ & 1.5 & 318.0 & $4 \cdot 1$ \\
\hline \multicolumn{18}{|l|}{ Survey phase } \\
\hline IV (2007-2009) & 16187 & $60 \cdot 0^{\star *}$ & 0.9 & 0.9 & 0.0 & $16 \cdot 1^{\star \star \star}$ & 1.0 & $48 \cdot 0$ & $2 \cdot 0$ & 37.5 & 1.7 & $62 \cdot 4^{\star \star \star}$ & 1.3 & $73 \cdot 8^{\star \star}$ & $2 \cdot 3$ & $298 \cdot 6^{*}$ & $4 \cdot 8$ \\
\hline V (2010-2012) & 17394 & $68 \cdot 3$ & $1 \cdot 2$ & 1.0 & 0.1 & $51 \cdot 8$ & 4.0 & $54 \cdot 2$ & 2.5 & 36.5 & $1 \cdot 8$ & 53.5 & 1.2 & 68.4 & 1.9 & 333.6 & $6 \cdot 3$ \\
\hline \multicolumn{18}{|l|}{ Sex } \\
\hline Male & 13721 & 74.4 & $1 \cdot 1$ & $1 \cdot 0^{\star *}$ & 0.1 & $33 \cdot 0^{* * *}$ & $2 \cdot 8$ & $42 \cdot 6^{\star \star \star}$ & 1.9 & $33 \cdot 2^{\star \star \star}$ & $1 \cdot 3$ & $67 \cdot 2^{\star \star \star}$ & 1.3 & $67 \cdot 5^{\star \star \star}$ & $2 \cdot 0$ & $318 \cdot 7^{\star \star \star}$ & $5 \cdot 1$ \\
\hline Female & 19860 & $55 \cdot 0$ & 0.8 & 0.9 & 0.1 & 38.8 & $2 \cdot 3$ & $60 \cdot 0$ & $2 \cdot 1$ & 40.7 & $1 \cdot 6$ & 48.0 & 0.9 & 74.0 & $1 \cdot 7$ & $317 \cdot 3$ & 4.9 \\
\hline \multicolumn{18}{|l|}{ Age (years) } \\
\hline $19-29$ & 3957 & $56 \cdot 8^{* \star \star}$ & 1.4 & $0.9^{\star \star \star}$ & 0.2 & $34 \cdot 2$ & 3.4 & $34 \cdot 5^{\star \star \star}$ & $2 \cdot 3$ & $25 \cdot 4^{\star \star \star}$ & 1.6 & $47 \cdot 8^{\star \star \star *}$ & 1.7 & $56 \cdot 2^{\star \star \star}$ & $2 \cdot 7$ & $255 \cdot 7^{\star \star \star}$ & $6 \cdot 6$ \\
\hline $30-49$ & 12553 & $67 \cdot 8$ & $1 \cdot 1$ & $1 \cdot 1$ & 0.1 & $39 \cdot 4$ & $3 \cdot 1$ & $57 \cdot 7$ & $2 \cdot 6$ & $37 \cdot 0$ & 1.5 & 64.9 & 1.3 & 73.5 & $2 \cdot \cdot 1$ & $341 \cdot 2$ & $6 \cdot 0$ \\
\hline $50-64$ & 8819 & 70.9 & 1.6 & 1.0 & 0.0 & $36 \cdot 6$ & 2.9 & $63 \cdot 3$ & 3.4 & $49 \cdot 3$ & $3 \cdot 1$ & $60 \cdot 1$ & 1.6 & $86 \cdot 1$ & $2 \cdot 8$ & $367 \cdot 3$ & $7 \cdot 8$ \\
\hline $65-74$ & 5434 & 60.9 & 1.9 & 0.7 & 0.0 & 28.5 & 3.4 & 43.4 & $2 \cdot 7$ & $36 \cdot 7$ & 1.9 & $45 \cdot 6$ & 1.5 & $68 \cdot 6$ & 2.9 & 284.3 & $7 \cdot 3$ \\
\hline $75+$ & 2818 & 46.5 & 1.9 & 0.5 & 0.0 & $22 \cdot 1$ & $3 \cdot 2$ & $24 \cdot 2$ & $2 \cdot 0$ & $26 \cdot 0$ & $2 \cdot 0$ & $40 \cdot 3$ & $2 \cdot 0$ & $40 \cdot 0$ & $2 \cdot 3$ & $199 \cdot 6$ & $6 \cdot 7$ \\
\hline \multicolumn{18}{|l|}{ Household income† } \\
\hline Low & 6964 & 55.9 & 1.5 & $0.6^{\star \star \star}$ & 0.0 & $21 \cdot 0^{\star \star *}$ & $2 \cdot 1$ & $32 \cdot 0^{\star \star *}$ & $2 \cdot 2$ & $31 \cdot 6$ & $2 \cdot 1$ & $46 \cdot 0^{\star \star \star}$ & 1.6 & $54 \cdot 4^{\star \star \star}$ & $3 \cdot 1$ & $241 \cdot 3^{\star \star \star}$ & $6 \cdot 5$ \\
\hline Middle low & 8383 & $62 \cdot 3$ & 1.5 & 0.8 & 0.0 & $39 \cdot 8$ & 4.0 & $44 \cdot 0$ & $2 \cdot 3$ & $35 \cdot 1$ & $2 \cdot 3$ & 55.5 & 1.6 & $67 \cdot 6$ & $2 \cdot 7$ & 304.9 & $7 \cdot 1$ \\
\hline Middle high & 8761 & 67.4 & 1.5 & $1 \cdot 2$ & 0.2 & $37 \cdot 3$ & 2.9 & $61 \cdot 1$ & 3.7 & 39.5 & 1.8 & 61.0 & 1.6 & 73.5 & $2 \cdot 4$ & 340.7 & $7 \cdot 2$ \\
\hline High & 8788 & $69 \cdot 7$ & 1.4 & $1 \cdot 1$ & 0.1 & $40 \cdot 1$ & $4 \cdot 1$ & 61.0 & 2.9 & $39 \cdot 9$ & 1.9 & $61 \cdot 7$ & 1.6 & 82.4 & $2 \cdot 8$ & $355 \cdot 6$ & $7 \cdot 5$ \\
\hline Education level & & & & & & & & & & & & & & & & & \\
\hline$\leqq$ Elementary & 8862 & $56 \cdot 8^{*}$ & 1.4 & $0.7^{\star \star \star}$ & 0.0 & $25 \cdot 4^{\star \star}$ & $2 \cdot 6$ & $37 \cdot 8^{\star \star \star}$ & $2 \cdot 3$ & $36 \cdot 1^{*}$ & $2 \cdot 1$ & $45 \cdot 4^{\star \star \star}$ & 1.4 & $62 \cdot 5^{\star \star}$ & $2 \cdot 8$ & $264 \cdot 7^{\star \star \star}$ & $6 \cdot 4$ \\
\hline Middle school & 3481 & 64.9 & $2 \cdot 1$ & 0.8 & 0.1 & $37 \cdot 8$ & 4.0 & $55 \cdot 0$ & 3.8 & $43 \cdot 8$ & $3 \cdot 8$ & 58.5 & $2 \cdot 6$ & 74.9 & $3 \cdot 6$ & 335.7 & $10 \cdot 4$ \\
\hline High school & 10262 & $64 \cdot 3$ & $1 \cdot 2$ & 1.0 & 0.2 & $38 \cdot 8$ & 3.6 & $50 \cdot 1$ & $2 \cdot 1$ & $36 \cdot 0$ & 1.5 & $57 \cdot 8$ & 1.4 & 68.4 & $2 \cdot 1$ & $316 \cdot 2$ & $6 \cdot 2$ \\
\hline$\geqq$ College & 8303 & $69 \cdot 7$ & 1.4 & $1 \cdot 1$ & 0.1 & $39 \cdot 7$ & 3.0 & 63.9 & $3 \cdot 1$ & $39 \cdot 0$ & $1 \cdot 8$ & 63.8 & 1.5 & $80 \cdot 1$ & 2.5 & $357 \cdot 1$ & $6 \cdot 9$ \\
\hline $\begin{array}{c}\text { Regular alcohol } \\
\text { consumption } \ddagger\end{array}$ & & & & & & & & & & & & & & & & & \\
\hline Yes & 15296 & $58 \cdot 8$ & 1.0 & 0.9 & 0.0 & $41 \cdot 4^{\star \star \star}$ & $3 \cdot 1$ & $60 \cdot 4^{\star \star \star}$ & $2 \cdot 3$ & $44 \cdot 0^{\star \star \star}$ & 2.4 & $52 \cdot 0$ & $1 \cdot 1$ & $79 \cdot 2^{\star \star \star}$ & 2.4 & $336 \cdot 6^{\star \star \star}$ & $6 \cdot 3$ \\
\hline No & 15564 & $68 \cdot 2$ & 1.0 & $1 \cdot 1$ & 0.1 & 31.8 & $2 \cdot 3$ & 44.9 & 1.8 & $32 \cdot 8$ & 1.2 & 61.6 & $1 \cdot 2$ & $66 \cdot 3$ & 1.8 & 306.5 & $4 \cdot 7$ \\
\hline Current smoking§ & & & & & & & & & & & & & & & & & \\
\hline Yes & 25039 & $62 \cdot 5^{\text {***}}$ & 0.8 & $1 \cdot 0^{*}$ & 0.1 & $40 \cdot 2^{\star \star \star}$ & 2.7 & $58 \cdot 2^{\star \star \star}$ & 1.9 & $40 \cdot 9^{\star \star \star}$ & $1 \cdot 6$ & $55 \cdot 2$ & 0.9 & $77 \cdot 0^{\star \star \star}$ & $1 \cdot 7$ & $334 \cdot 7^{\star \star \star}$ & 4.9 \\
\hline No & 5878 & $69 \cdot 5$ & 1.6 & 0.9 & 0.0 & $23 \cdot 1$ & $2 \cdot 1$ & $31 \cdot 7$ & $2 \cdot 0$ & $27 \cdot 8$ & 1.7 & $64 \cdot 3$ & 1.9 & $57 \cdot 5$ & $2 \cdot 8$ & $273 \cdot 6$ & $6 \cdot 2$ \\
\hline Physical activityll & & & & & & & & & & & & & & & & & \\
\hline Active & 26462 & $62 \cdot 8^{*}$ & 0.8 & $1 \cdot 0$ & 0.1 & $36 \cdot 2$ & $2 \cdot 3$ & $50 \cdot 7$ & $1 \cdot 7$ & $37 \cdot 5$ & 1.6 & $57 \cdot 0$ & 0.9 & $70 \cdot 6$ & $1 \cdot 7$ & 315.5 & $4 \cdot 6$ \\
\hline Inactive & 4415 & $72 \cdot 3$ & 1.9 & $1 \cdot 0$ & 0.1 & $34 \cdot 2$ & 3.9 & $56 \cdot 4$ & $3 \cdot 2$ & $27 \cdot 9$ & $2 \cdot 1$ & $60 \cdot 4$ & $2 \cdot 0$ & $78 \cdot 0$ & 3.5 & $340 \cdot 1$ & $8 \cdot 4$ \\
\hline Supplement useף & & & & & & & & & & & & & & & & & \\
\hline Yes & 10008 & $69 \cdot 9^{\star \star \star}$ & 1.5 & $1 \cdot 1^{*}$ & 0.1 & $43 \cdot 3^{\star \star}$ & $3 \cdot 1$ & $63 \cdot 5^{\star \star \star}$ & $3 \cdot 1$ & $44 \cdot 1^{\star \star \star}$ & $2 \cdot 1$ & 55.9 & 1.4 & $83 \cdot 6^{\star \star \star}$ & $2 \cdot 4$ & $361 \cdot 2^{\star \star \star}$ & $6 \cdot 8$ \\
\hline No & 23194 & 62.5 & 0.8 & 0.9 & 0.1 & $33 \cdot 0$ & $2 \cdot 3$ & $46 \cdot 3$ & 1.7 & 34.0 & 1.3 & $58 \cdot 1$ & 1.0 & $65 \cdot 3$ & 1.6 & $299 \cdot 8$ & 4.4 \\
\hline $\begin{array}{l}\text { Breakfast } \\
\text { consumption†† }\end{array}$ & & & & & & & & & & & & & & & & & \\
\hline Yes & 26689 & $57 \cdot 1^{\star * *}$ & $1 \cdot 1$ & $0.8^{*}$ & 0.0 & $37 \cdot 1$ & 3.4 & $38 \cdot 7^{\star \star \star}$ & $2 \cdot 0$ & $28 \cdot 5^{\star \star \star}$ & 1.5 & $49 \cdot 9^{\star \star \star}$ & 1.4 & $58 \cdot 9^{\star \star \star}$ & $2 \cdot 4$ & $270 \cdot 7^{\star \star \star}$ & $6 \cdot 3$ \\
\hline No & 6874 & $67 \cdot 4$ & 0.9 & $1 \cdot 0$ & 0.1 & 35.5 & $2 \cdot 3$ & $56 \cdot 0$ & $2 \cdot 0$ & $40 \cdot 0$ & 1.5 & $60 \cdot 2$ & 1.0 & $75 \cdot 1$ & $1 \cdot 7$ & $335 \cdot 1$ & $4 \cdot 7$ \\
\hline Eating out & & & & & & & & & & & & & & & & & \\
\hline$\geqq 1$ time/d & 6498 & $60 \cdot 5$ & $1 \cdot 8$ & 0.8 & 0.1 & $46 \cdot 5^{\star}$ & 6.5 & $53 \cdot 0^{\star \star *}$ & 3.8 & $39 \cdot 3^{\star \star \star}$ & $2 \cdot 5$ & $44 \cdot 8^{* * *}$ & 1.7 & $71 \cdot 2^{\star \star \star}$ & 2.9 & $316 \cdot 0^{\star \star \star}$ & $9 \cdot 7$ \\
\hline$\overline{1}-6$ times/week & 20792 & 61.9 & 0.9 & 1.0 & 0.1 & $33 \cdot 1$ & 2.0 & $54 \cdot 3$ & $2 \cdot 0$ & $37 \cdot 7$ & 1.3 & $57 \cdot 4$ & $1 \cdot 1$ & $73 \cdot 3$ & 1.8 & 318.5 & 4.6 \\
\hline$\leqq 3$ time/month & 6268 & $73 \cdot 4$ & 1.6 & 1.0 & 0.0 & $35 \cdot 5$ & $3 \cdot 2$ & $44 \cdot 1$ & $2 \cdot 7$ & 33.9 & $2 \cdot 3$ & $66 \cdot 0$ & $1 \cdot 7$ & $65 \cdot 1$ & $2 \cdot 4$ & $318 \cdot 7$ & $7 \cdot 0$ \\
\hline Foōd Securitył‡ & & & & & & & & & & & & & & & & & \\
\hline Full & 14038 & 66.9 & $1 \cdot 1$ & $1 \cdot 1^{\star \star \star}$ & 0.0 & $38 \cdot 3^{\star \star \star}$ & 2.7 & $56 \cdot 1^{\star \star \star}$ & $2 \cdot 1$ & $39 \cdot 8^{\star *}$ & 1.7 & $61 \cdot 0^{*}$ & $1 \cdot 3$ & $76 \cdot 5^{\star \star \star}$ & $2 \cdot 2$ & $339 \cdot 3^{\star \star \star}$ & 5.5 \\
\hline Marginal & 17362 & $64 \cdot 2$ & $1 \cdot 1$ & 0.9 & 0.1 & 35.9 & 2.7 & $50 \cdot 7$ & $2 \cdot 3$ & $36 \cdot 0$ & 1.5 & $56 \cdot 0$ & 1.2 & 68.9 & 1.9 & 312.5 & $5 \cdot 4$ \\
\hline Low & 1750 & 52.5 & 2.5 & 0.6 & 0.0 & 20.5 & $4 \cdot 1$ & $24 \cdot 4$ & 2.9 & $25 \cdot 8$ & $2 \cdot 8$ & $44 \cdot 0$ & $3 \cdot 1$ & $50 \cdot 1$ & $4 \cdot 3$ & $217 \cdot 8$ & $9 \cdot 3$ \\
\hline Very low & 377 & $48 \cdot 4$ & $6 \cdot 7$ & 0.4 & 0.1 & $9 \cdot 4$ & $2 \cdot 7$ & $23 \cdot 1$ & $6 \cdot 1$ & $22 \cdot 2$ & $4 \cdot 1$ & $48 \cdot 8$ & $6 \cdot 6$ & 35.7 & $6 \cdot 8$ & 188.0 & $18 \cdot 7$ \\
\hline
\end{tabular}

$P$ values for the differences by the $t$ tests and ANOVA among the subgroups after adjusting for total energy intake ${ }^{*} P<0.05,{ }^{\star *} P<0.01,{ }^{\star \star \star} P<0.001$.

$\dagger$ Household income: low (first quartile), middle-low (second quartile), middle-high (third quartile), high (fourth quartile).

$\ddagger$ Regular alcohol drinking: 'yes' meant that the person drank more than once a month over the past year.

$\S$ Current smoking: 'yes' meant that the person smoked >100 cigarettes over his or her lifetime and still smokes.

॥ Physical activity: 'active' meant that the person performed vigorous-intensity physical activity, which requires a large amount of effort and causes rapid breathing for $>20$ min once for $>3 \mathrm{~d} /$ week.

II Supplement use: 'yes' meant taking dietary supplements.

†† Breakfast consumption: 'yes' meant that the person had breakfasts on both the days before the interview.

ł‡ Food security: 'full' meant able to eat an adequate amount and variety of food, 'marginal' meant able to eat an adequate amount but not variety of food, 'low' meant sometimes unable to afford enough food, 'very low' meant often unable to afford enough food. 
contributors to the intake of isoflavones. Proanthocyanidins were obtained mainly from fruits, grains and legumes and legume products.

The main individual food contributors to flavonoid intake were also analysed (Table 4). We identified apples (21.9\%), mandarins $(12.5 \%)$, tofu $(11.5 \%)$, onions (9.6\%) and grapes $(9.0 \%)$ as the major sources of the total flavonoids. For flavonols, onions $(49.2 \%)$, radish leaves $(14.4 \%)$, radishes (9.7\%), soyabeans $(6.1 \%)$ and apples $(5.5 \%)$ were the main contributors. The most abundant sources of flavones were chili

Table 2. Composition of the individual flavonoid intakes among Korean adults

\begin{tabular}{|c|c|c|c|}
\hline Subclasses and compounds & Intake $(\mathrm{mg} / \mathrm{d})$ & $\%$ of subclass & $\%$ of total \\
\hline Flavonols & $64 \cdot 6$ & & $20 \cdot 3$ \\
\hline Quercetin & 48.9 & $75 \cdot 6$ & $15 \cdot 4$ \\
\hline Kaempferol & $12 \cdot 8$ & $19 \cdot 9$ & $4 \cdot 0$ \\
\hline Myricetin & 0.8 & 1.2 & 0.3 \\
\hline Isorhamnetin & $2 \cdot 1$ & 3.3 & 0.7 \\
\hline Flavones & 1.0 & & 0.3 \\
\hline Luteolin & 0.7 & $77 \cdot 1$ & 0.2 \\
\hline Apigenin & 0.2 & 21.9 & 0.1 \\
\hline Flavanones & 35.9 & & $11 \cdot 3$ \\
\hline Hesperetin & $28 \cdot 1$ & 78.4 & 8.8 \\
\hline Naringenin & 7.5 & $21 \cdot 0$ & $2 \cdot 4$ \\
\hline Eriodictyol & 0.2 & 0.6 & 0.1 \\
\hline Flavan-3-ols & 51.4 & & $16 \cdot 2$ \\
\hline Catechin & $10 \cdot 1$ & $19 \cdot 6$ & $3 \cdot 2$ \\
\hline Epicatechin & $7 \cdot 1$ & $13 \cdot 7$ & $2 \cdot 2$ \\
\hline Epigallocatechin & 33.4 & 64.9 & 10.5 \\
\hline Theaflavin & 0.1 & 0.2 & 0.0 \\
\hline Theaflavin-3-gallate & $0 \cdot 1$ & 0.2 & 0.0 \\
\hline Theaflavin-3'-gallate & 0.0 & 0.0 & 0.0 \\
\hline Theaflavin-3,3'-digallate & 0.0 & 0.0 & 0.0 \\
\hline Thearubigin & 0.6 & 1.2 & 0.2 \\
\hline Anthocyanidins & $37 \cdot 0$ & & 11.6 \\
\hline Cyanidin & $13 \cdot 0$ & $35 \cdot 2$ & $4 \cdot 1$ \\
\hline Delphinidin & 9.5 & $25 \cdot 6$ & 3.0 \\
\hline Malvidin & $2 \cdot 4$ & 6.4 & 0.7 \\
\hline Pelargonidin & $8 \cdot 0$ & $21 \cdot 6$ & 2.5 \\
\hline Peonidin & 1.8 & 4.8 & 0.6 \\
\hline Petunidin & $2 \cdot 3$ & $6 \cdot 3$ & 0.7 \\
\hline Isoflavones & 57.5 & & $18 \cdot 1$ \\
\hline Daidzein & $20 \cdot 4$ & 35.4 & $6 \cdot 4$ \\
\hline Genistein & 28.5 & 49.5 & 8.9 \\
\hline Glycetin & 8.6 & $15 \cdot 0$ & $2 \cdot 7$ \\
\hline Proanthocyanidins & $70 \cdot 8$ & & $22 \cdot 3$ \\
\hline Dimers & $10 \cdot 7$ & $15 \cdot 1$ & 3.4 \\
\hline Trimers & $5 \cdot 0$ & $7 \cdot 0$ & 1.6 \\
\hline 4-6 monomers & 14.4 & $20 \cdot 3$ & 4.5 \\
\hline 7-10 monomers & $10 \cdot 2$ & $14 \cdot 3$ & 3.2 \\
\hline Polymers & $30 \cdot 7$ & $43 \cdot 3$ & $9 \cdot 6$ \\
\hline
\end{tabular}

peppers $(33.8 \%)$, sweet peppers $(12 \cdot 1 \%)$, watermelons (9.0 \%), parsley ( $8.5 \%)$ and perilla seeds ( $8.3 \%)$. Mandarins (90.3\%) and oranges $(6.5 \%)$ were the exclusive sources of flavanones, and apples $(84.9 \%)$ and green tea $(7.6 \%)$ were the major sources of flavan-3-ols. Grapes (37.6\%), persimmons $(24.3 \%)$ and radishes $(16.4 \%)$ were the prime sources of anthocyanidins, and apples (27.8\%), grapes (17.7\%) and sorghum (12.7\%) were the major sources of proanthocyanidins. We identified tofu $(66.7 \%)$ as the richest contributor of proanthocyanidins, followed by mandarins (11.7\%), soyabean sprouts (11.6\%), soyabean paste $(4.0 \%)$ and soyabeans $(2 \cdot 1 \%)$.

In the multiple regression analysis, intakes of legumes and legume products, vegetables and fruits were major predictors of total flavonoid intake. Regression estimates indicated that one serving of legumes and legume products, vegetables and fruits per $\mathrm{d}$ could increase the total flavonoid intake by $82.31 \mathrm{mg} / \mathrm{d}(P<0.0001), 3.94 \mathrm{mg} / \mathrm{d}(P=0.0086)$ and $78.30 \mathrm{mg} / \mathrm{d}$ $(P<0.0001)$, respectively. The consumptions of legumes and legume products, vegetables, fruits and beverage and alcohols were significant predictors of flavonol intake; those of legumes and legume products, vegetables and fruits were strong predictors of isoflavone intake; and those of legumes and legume products and fruits were strong predictors of proanthocyanidin intake. In the model for anthocyanidins intake, fruits consumption was the only significant predictor (Table 5).

\section{Discussion}

As only a few studies on flavonoid intakes have focused on the Korean population, we investigated the epidemiological characteristics of flavonoid intakes in Korean adults. We constructed a new KFDB and found that the mean daily total flavonoid intake of Korean adults was $318.0 \mathrm{mg} / \mathrm{d}$, and the major food sources were fruits, vegetables and legumes and legume products.

A comprehensive database is an essential part in epidemiological studies for estimating flavonoid intake ${ }^{(26)}$. The Korea Functional Food Composition Table, published by the National Academy of Agricultural Science, is the only currently available phytochemical database for Korean common foods, but it covers a limited number of food items ${ }^{(40)}$. To fill the gap, we constructed a new flavonoid database for common Korean foods that appeared in the KNHANES (2007-2012) food consumption data. The new flavonoid database covered $49 \%$ of the food items and $76 \%$ of the food intake for thirty-one individual

Table 3. Contribution (\%) of food groups to flavonoid intakes among Korean adults

\begin{tabular}{lcccccrrr}
\hline Food groups & Flavonols & Flavones & Flavanones & Flavan-3-ols & Anthocyanidins & Isoflavones & Proanthocyanidins & Total flavonoids \\
\hline Grains & 0.0 & 0.4 & 0.0 & 0.4 & 2.5 & 0.1 & 18.3 & 4.5 \\
Legumes and legume products & 6.2 & 0.8 & 0.0 & 0.4 & 5.1 & 70.3 & 9.9 & 16.2 \\
Nuts and seeds & 0.0 & 8.3 & 0.0 & 0.0 & 0.0 & 0.0 & 0.8 & 0.2 \\
Vegetables & 82.6 & 67.4 & 0.2 & 0.1 & 17.6 & 12.1 & 0.0 & 20.5 \\
Fruits & 8.5 & 20.9 & 99.7 & 89.1 & 74.4 & 11.9 & 67.4 & 54.4 \\
Beverages and alcohols & 2.7 & 2.0 & 0.1 & 9.8 & 0.5 & 1.0 & 3.0 & 0.1 \\
Seasonings & 0.0 & 0.0 & 0.0 & 0.0 & 0.0 & 4.6 & 0.1 & 0.0 \\
Others & 0.0 & 0.2 & 0.0 & 0.2 & 0.0 & 0.0 & 0.5 \\
\hline
\end{tabular}


Table 4. Top five food sources contributing to the flavonoid intake among Korean adults

\begin{tabular}{|c|c|c|c|c|c|}
\hline Compounds & Food item & Concentrations $(\mathrm{mg} / 100 \mathrm{~g})$ & Intake (mg/d) & Contribution (\%) & Cum $(\%)^{*}$ \\
\hline \multirow[t]{5}{*}{ Total flavonoids } & Apples & $102 \cdot 0$ & $70 \cdot 3$ & 21.9 & 21.9 \\
\hline & Mandarins & 224.5 & $40 \cdot 1$ & 12.5 & 34.4 \\
\hline & Tofu & 99.1 & $36 \cdot 8$ & 11.5 & $45 \cdot 9$ \\
\hline & Onions & $26 \cdot 7$ & $30 \cdot 8$ & 9.6 & 55.5 \\
\hline & Grapes & $129 \cdot 2$ & $28 \cdot 7$ & $9 \cdot 0$ & 64.5 \\
\hline \multirow{5}{*}{ Flavonols } & Onions & $26 \cdot 7$ & $30 \cdot 8$ & $49 \cdot 2$ & $49 \cdot 2$ \\
\hline & Radish leaves & $43 \cdot 7$ & $9 \cdot 0$ & 14.4 & $63 \cdot 6$ \\
\hline & Radishest & 21.0 & $6 \cdot 1$ & $9 \cdot 7$ & $73 \cdot 3$ \\
\hline & Soyabeans & $155 \cdot 4$ & 3.8 & $6 \cdot 1$ & $79 \cdot 1$ \\
\hline & Apples & 10.9 & 3.4 & 5.5 & 84.9 \\
\hline \multirow[t]{5}{*}{ Flavones } & Chili peppers & 3.1 & 0.3 & 33.8 & 33.8 \\
\hline & Sweet peppers & $12 \cdot 8$ & $0 \cdot 1$ & $12 \cdot 1$ & $45 \cdot 9$ \\
\hline & Watermelons & 0.5 & $0 \cdot 1$ & $9 \cdot 0$ & 54.9 \\
\hline & Parsley $\ddagger$ & $2622 \cdot 1^{*}$ & $0 \cdot 1$ & 8.5 & $63 \cdot 4$ \\
\hline & Perilla seeds & $15 \cdot 7$ & 0.1 & 8.3 & $71 \cdot 7$ \\
\hline \multirow[t]{5}{*}{ Flavanones } & Mandarins & 210.9 & $32 \cdot 6$ & $90 \cdot 3$ & $90 \cdot 3$ \\
\hline & Oranges & $21 \cdot 4$ & $2 \cdot 4$ & 6.5 & $96 \cdot 8$ \\
\hline & Fruit drinks§ & $2.8 \dagger$ & 0.7 & 1.8 & 98.6 \\
\hline & Grapefruits & $67 \cdot 2$ & 0.2 & 0.6 & $99 \cdot 2$ \\
\hline & Kumquats & $41 \cdot 0$ & 0.1 & 0.4 & $99 \cdot 6$ \\
\hline \multirow[t]{5}{*}{ Flavan-3-ols } & Apples & 29.8 & $46 \cdot 3$ & 84.9 & 84.9 \\
\hline & Green teall & 1044.1‡ & $4 \cdot 2$ & $7 \cdot .6$ & 92.5 \\
\hline & Grapes & 1.6 & 0.9 & 1.7 & 94.2 \\
\hline & Black teaף & $308 \cdot 7 \S$ & 0.8 & 1.4 & $95 \cdot 6$ \\
\hline & Strawberries & 8.6 & 0.4 & 0.8 & $96 \cdot 4$ \\
\hline \multirow[t]{5}{*}{ Anthocyanidins } & Grapes & $17 \cdot 0$ & 14.5 & $37 \cdot 6$ & $37 \cdot 6$ \\
\hline & Persimmons & $53 \cdot 3$ & 9.4 & $24 \cdot 3$ & 61.9 \\
\hline & Radishes & $62 \cdot 2$ & $6 \cdot 4$ & $16 \cdot 4$ & $78 \cdot 3$ \\
\hline & Strawberries & $57 \cdot 6$ & $2 \cdot 9$ & 7.5 & $85 \cdot 8$ \\
\hline & Soyabeans & 4.1 & 1.5 & 3.9 & $89 \cdot 7$ \\
\hline \multirow[t]{5}{*}{ Isoflavones } & Tofu & $99 \cdot 1$ & $36 \cdot 8$ & $66 \cdot 7$ & $66 \cdot 7$ \\
\hline & Mandarins & 3.7 & $6 \cdot 4$ & $11 \cdot 7$ & 78.4 \\
\hline & Soyabean sprouts & 48.9 & $6 \cdot 4$ & 11.6 & $90 \cdot 0$ \\
\hline & Soyabean paste & $20 \cdot 9$ & $2 \cdot 2$ & 4.0 & $94 \cdot 0$ \\
\hline & Soyabeans & $36 \cdot 9$ & 1.2 & $2 \cdot 1$ & $96 \cdot 1$ \\
\hline \multirow[t]{5}{*}{ Proanthocyanidins } & Apples & $60 \cdot 4$ & $20 \cdot 3$ & $27 \cdot 8$ & $27 \cdot 8$ \\
\hline & Grapes & $109 \cdot 3$ & $12 \cdot 9$ & $17 \cdot 7$ & 40.5 \\
\hline & Sorghum & 1951.0 & $9 \cdot 2$ & $12 \cdot 7$ & $53 \cdot 2$ \\
\hline & Strawberries & $140 \cdot 8$ & $7 \cdot 1$ & $9 \cdot 7$ & $62 \cdot 9$ \\
\hline & Peaches & 30.5 & 4.3 & 5.9 & $68 \cdot 8$ \\
\hline
\end{tabular}

* Cumulative percentage of the food item contributing to flavonoid subclass and total flavonoid intakes.

$\dagger$ Includes radish, raw; radish, dried; radish, pickled; red beet; turnip.

$\ddagger$ Includes parsley, raw; parsley, dried.

$\S$ Includes fruit juice; fruitade; fruit nectar; fruit-flavoured drink.

II Includes green tea, powder; green tea, leaves; green tea, infusion.

II Includes black tea, tea bag; black tea, infusion; black tea, flavoured.

flavonoids in seven flavonoid subclasses, which could enhance the accuracy of flavonoid intake estimation.

The mean daily intake of total flavonoids by Korean adults was $318.0 \mathrm{mg}$, which was slightly lower compared with other countries. The mean intake of total flavonoids by US adults was estimated to range from 189.8 to $207.30 \mathrm{mg} / \mathrm{d}$ based on six of the seven subclasses of flavonoids, excluding proanthocyanidins ${ }^{(35,43)}$, and $344.83 \mathrm{mg} / \mathrm{d}$ based on all seven subclasses ${ }^{(36)}$. European adults in the European Prospective Investigation into Cancer and Nutrition (EPIC) cohort had a mean intake of $370 \cdot 2-437 \cdot 2 \mathrm{mg} / \mathrm{d}$ when seven subclasses except for thearubigins were included ${ }^{(44,45)}$; the Spanish cohort had an intake of $376.69 \mathrm{mg} / \mathrm{d}$ when all seven subclasses and their thirty-five individual flavonoids were included ${ }^{(46)}$. In a sample of Chinese adults, the average daily intake of total flavonoids was estimated to be $165.6 \mathrm{mg} / \mathrm{d}$ from fruit, vegetable and nut intakes for anthocyanidins, flavonols, flavones and isoflavones ${ }^{(47)}$. The differences in these amounts could be due to the differences in diet among the populations, dietary data collection methods, the extent of flavonoid components included in the databases, cultivars and geographical features of the food sources and other factors.

In the present study, total flavonoid intake was significantly higher among females, especially in the 30-64-year-old group, subjects with higher household incomes and higher education level, non-regular alcohol drinkers, non-current smokers, those who take supplements, those who eat breakfast regularly and those with higher food security, after adjusting for total energy intake. Distinct distributions by sex and age can be explained by the findings of a previous study, which reported that the percentages of Korean adults consuming the recommended daily fruit and vegetable intakes were higher among females 
Table 5. Regression estimates of food groups for flavonoid intakes among Korean adults ( $\beta$ Coefficients and coefficient of determination)

\begin{tabular}{|c|c|c|c|}
\hline Variables ${ }^{*}, \dagger$ & Slope $(\beta)$ & $P$ & Model $R^{2} \ddagger$ \\
\hline \multicolumn{4}{|l|}{ Total flavonoids $(\mathrm{mg} / \mathrm{d})$} \\
\hline Legumes and legume products consumption (serving§) & $82 \cdot 31$ & $<0.0001$ & \multirow[t]{4}{*}{0.333} \\
\hline Vegetables consumption (serving) & 3.94 & 0.0086 & \\
\hline Fruits consumption (serving) & $78 \cdot 30$ & $<0.0001$ & \\
\hline Beverage and alcohols consumption (serving) & 0.26 & 0.8524 & \\
\hline \multicolumn{4}{|l|}{ Flavonols (mg/d) } \\
\hline Legumes and legume products consumption (serving) & 14.48 & $<0.0001$ & \multirow[t]{4}{*}{0.160} \\
\hline Vegetables consumption (serving) & $5 \cdot 20$ & $<0.0001$ & \\
\hline Fruits consumption (serving) & 2.52 & $<0.0001$ & \\
\hline Beverage and alcohols consumption (serving) & 0.68 & 0.0190 & \\
\hline \multicolumn{4}{|l|}{ Anthocyanidins (mg/d) } \\
\hline Legumes and legume products consumption (serving) & -0.80 & 0.6352 & \multirow[t]{4}{*}{0.247} \\
\hline Vegetables consumption (serving) & -0.80 & 0.1314 & \\
\hline Fruits consumption (serving) & 21.98 & 0.0002 & \\
\hline Beverage and alcohols consumption (serving) & -0.61 & 0.0977 & \\
\hline \multicolumn{4}{|l|}{ Isoflavones (mg/d) } \\
\hline Legumes and legume products consumption (serving) & 46.07 & $<0.0001$ & \multirow[t]{4}{*}{0.282} \\
\hline Vegetables consumption (serving) & 1.79 & $<0.0001$ & \\
\hline Fruits consumption (serving) & 1.71 & 0.0006 & \\
\hline Beverage and alcohols consumption (serving) & 0.03 & 0.9166 & \\
\hline \multicolumn{4}{|l|}{ Proanthocyanidins (mg/d) } \\
\hline Legumes and legume products consumption (serving) & 21.00 & $<0.0001$ & \multirow[t]{4}{*}{0.179} \\
\hline Vegetables consumption (serving) & -0.87 & 0.1173 & \\
\hline Fruits consumption (serving) & $22 \cdot 27$ & $<0.0001$ & \\
\hline Beverage and alcohols consumption (serving) & -0.03 & 0.9328 & \\
\hline
\end{tabular}

* Adjusted by survey phase, sex, household income, education level, regular alcohol drinking, current smoking, supplement use, breakfast consumption, food security and total energy intake. † Flavones, flavanones and flavonols in which model $R^{2}$ was $<0.150$ are not shown.

$\ddagger P$ values for all the models were $<0.0001$.

$\S$ Servings of each food group was calculated according to the Korean Food Guidance System.

than in males and were higher in the $\geqq 40$-year-old group than in the other groups ${ }^{(38)}$. The older adults might adhere more to a traditional Korean diet, which is mainly composed of plant food $^{(48)}$ and pay more attention to health care. People with a lower socio-economic status, as characterised by a low income level, low education level and low food security, were consistently reported to consume less amounts of flavonoids ${ }^{(10,37,46)}$, which seem to be attributable to lower amounts of fruits and vegetables ${ }^{(49-51)}$. Moreover, unhealthy lifestyles that included regular alcohol drinking, current smoking, irregular physical activity and infrequent breakfast consumption also resulted in an inadequate intake of flavonoid-high food items such as fruits and vegetables ${ }^{(50-53)}$. The significant difference in flavonoid intake between supplement users and non-users in our study was also shown by other researchers ${ }^{(35,43)}$. The prevalence of chronic diseases may be influenced by these socio-demographic and lifestyle factors through the mediation of different flavonoid intakes.

The top five food items contributing to the total flavonoid intake in Korean adults were apples, mandarins, tofu, onions and grapes. A US study reported that the major food sources of daily flavonoid intakes were tea, wines, beers, citrus fruits and apples ${ }^{(36)}$, and the EPIC study also reported that fruits, tea, wines and fruit juices were the main food sources of total flavonoid intake $e^{(44)}$. As soyabean products such as soyabean paste and tofu are part of the traditional Korean diet, legumes and legume products contributed far more to the total flavonoid intake in the Korean population compared with that of the US and European populations, whereas tea and wines contributed far less in Koreans.
In addition to the differences in total flavonoid intake, the differences in flavonoid compositions have been observed across studies. In this study, proanthocyanidins $(22.3 \%$, $70 \cdot 8 \mathrm{mg} / \mathrm{d}$ ) contributed the most to the total daily flavonoid intake, followed by flavonols $(20.3 \%, 64.6 \mathrm{mg} / \mathrm{d})$, isoflavones $(18.1 \%, \quad 57.5 \mathrm{mg} / \mathrm{d})$ and flavan-3-ols $(20.5 \%, \quad 51.4 \mathrm{mg} / \mathrm{d})$. Zamora-Ros et al. ${ }^{(10)}$ reported similarly that proanthocyanidins $(44.0 \%, 182.7 \mathrm{mg} / \mathrm{d})$ were the primary contributors to the total flavonoid intake in EPIC-InterAct study participants, largely from fruits, wines, chocolate and juices. Among European countries, different amounts of proanthocyanidin intake were observed. The southern region, including France, Italy and Spain, consumed more proanthocyanidins than the Central and Northern regions ${ }^{(46,54,55)}$. On the other hand, Bai et al. ${ }^{(36)}$ reported proanthocyanidins as secondary contributors $(28.5,98.29 \mathrm{mg} / \mathrm{d})$ to the total flavonoid intake in US adults, largely from wines, legumes, tea, strawberries and plums. However, Korean adults consumed a large amount of proanthocyanidins from fruits, grains and legumes and legume products.

Several studies on flavonoid intake have reported that flavan3 -ols are the greatest contributors to the total flavonoid intake, $156.5 \mathrm{mg} / \mathrm{d}$ in the USA study with three flavan-3-ol monomers, theaflavins and thearubigins in their database ${ }^{(35,56)}$, and $182.4 \mathrm{mg} / \mathrm{d}$ in the EPIC study with seven monomers and theaflavins in their database ${ }^{(44)}$. In a study by Otaki et al. ${ }^{(57)}$ that did not include proanthocyanidins and anthocyanidins in their analysis, Japanese women obtained flavonoids mainly from flavan-3-ols, comprised of only three flavan-3-ol monomers 
(80.0\%, $1277 \mu \mathrm{mol} / \mathrm{d} ; 386.30 \mathrm{mg} / \mathrm{d}$ ). The flavan-3-ol intake level in the present study $(50.6 \mathrm{mg} / \mathrm{d})$ was very low compared with those in the studies of other populations. Our finding was supported by a study that estimated the daily flavan-3-ol intake for its twelve components from only plant food sources among Korean adults at $30.0 \mathrm{mg} / \mathrm{d}$ for men and $22.5 \mathrm{mg} / \mathrm{d}$ for women ${ }^{(37)}$, suggesting that Koreans consume a relatively small amount of flavan-3-ols. Considering that the major sources of flavan-3-ols in other countries are tea, fruits and wine, the low consumption of tea and red wine by Korean adults may be the reason for this. Koreans obtained flavan-3-ols mostly from apples ( $84.9 \%)$, followed by green tea, grapes, black tea and strawberries.

The largest difference has been found in the amount of isoflavone intake. Koreans consumed a lot more isoflavones than Western people. US and European people consume very small amounts of isoflavones, ranging from 0.10 to $2 \cdot 35 \mathrm{mg} / \mathrm{d}^{(10,35,36,44,46,58,59)}$, whereas Asians consume $6 \cdot 2-54 \cdot 3 \mathrm{mg} / \mathrm{d}^{(60,61)}$. Dietary patterns influenced by cultural habits might be attributable to this disparity. For example, Asians consume more soyabeans and soyabean products, which are abundant in isoflavones than Western people. In addition to soya foods, fruits, grains and coffee are good dietary sources of isoflavones ${ }^{(10,38)}$. We found that the major individual food sources of isoflavones in Korean adults were tofu (66.7\%) and mandarins $(11.7 \%)$, followed by soyabean sprouts, soyabean paste and soyabeans.

The estimated flavonol intake of $64.6 \mathrm{mg} / \mathrm{d}$ was higher than that in the USA $(12 \cdot 8-18 \cdot 1 \mathrm{mg} / \mathrm{d})^{(35,36)}$, Europe $(18 \cdot 7-51 \cdot 0 \mathrm{mg} / \mathrm{d})^{(10,44,46,62)}$, Japan $(58.4 \mu \mathrm{mol} / \mathrm{d}, 17.5 \mathrm{mg} / \mathrm{d})^{(57)}$ and China $(19.13 \mathrm{mg} / \mathrm{d})^{(63)}$. In accordance with these studies, which showed that flavonols are abundant in tea, fruits and vegetables, a significant amount of flavonol intake in our study was from vegetables and fruit. Meanwhile, flavones were the smallest contributors to total flavonoids, and the intake amount $(1.0 \mathrm{mg} / \mathrm{d})$ of Korean adults was smaller than that of the above-mentioned populations. This can be explained by the relatively lower flavone content and lower intake of its top five food sources. Yeon et al. ${ }^{(39)}$ found a lower estimated flavanone intake in Korean middle-aged men and women $(0.78 \mathrm{mg} / \mathrm{d})$ than ours; this difference appears to be due to the more comprehensive flavonoid databases used in the present study.

Korean adults in the present study had a flavanone intake level of $35.9 \mathrm{mg} / \mathrm{d}$. Flavanone intake was predominantly from fruits (99.7\%) such as mandarins, oranges, fruit drink, grapefruits and kumquats. The flavanone intakes of Finnish adults were estimated to be $31 \mathrm{mg} / \mathrm{d}^{(58)}$, of French adults $26 \mathrm{mg} / \mathrm{d}^{(62)}$, of Spanish adults $50.44 \mathrm{mg} / \mathrm{d}^{(46)}$ and of US adults $22.38 \mathrm{mg} / \mathrm{d}^{(36)}$. The anthocyanidin intake also varied among different studies. Finnish adults consumed the highest amount of anthocyanidins $(47 \mathrm{mg} / \mathrm{d})$, followed by Korean adults $(37.0 \mathrm{mg} / \mathrm{d})$ and Mediterranean people $(9-37 \cdot 42 \mathrm{mg} / \mathrm{d})^{(45,46,59,64)}$, whereas US adults consumed only $9 \cdot 20 \mathrm{mg} / \mathrm{d}^{(36,65)}$. The dietary sources of anthocyanidins in Korean adults were fruits, vegetables, especially grapes, persimmons, radishes and strawberries, as reported in other studies ${ }^{(36,38)}$, whereas legumes and legume products, such as soyabeans, were the unique contributors of anthocyanidin intake in Korean adults.
In our study, legumes and legume products, vegetables and fruits were strong predictors in the regression model of flavonoid intake by specific food group intake in Korean adults. If an individual consumes one more serving of legumes and legume products, vegetables and fruits per $\mathrm{d}$, he or she can expect to have $82.31,3.94$ and $78.03 \mathrm{mg}$ increase in the amount of total flavonoids per $\mathrm{d}$, respectively. The higher regression coefficient of legumes and legume products and fruits than that of vegetables may be partly due to the higher weight of one serving. For anthocyanidins, only fruits were significant independent predictors, and one serving of fruits may lead to an increase of $21.98 \mathrm{mg} / \mathrm{d}$ of anthocyanidin intake. These findings can provide basic information for developing dietary recommendations for flavonoid intakes.

In this study, the expanded database that focused on the foods commonly consumed by Koreans, with greater coverage, allowed us to estimate the flavonoid intakes and their dietary sources among Koreans. This database can also be used in future studies to elucidate the health benefits of dietary flavonoids. However, there are several limitations to this study. First, our results could be underestimated because of the limited coverage of food items in the KFDB and the exclusion of dietary supplements in our analysis. Second, the accuracy of some flavonoid contents in the KFDB might be lower than expected. For example, thearubigin contents that we included in our database is a crude estimation quantified by an indirect method $^{(66)}$. Nevertheless, the inclusion of thearubigins may not have much impact on the estimation of total flavonoids as tea, the only food contributor of thearubigins, is not highly consumed in our population. Finally, the use of a single 24-h recall might not be sufficient to reflect an individual's usual diet due to large intra-individual variability. Further research using more a comprehensive database and more accurate dietary assessment methods could improve the quality of our study.

Considering that the antioxidant capacity of flavonoids is reported to be related with health benefits ${ }^{(67)}$, construction of a database including a wider scope of antioxidant materials, such as antioxidant vitamins and carotenoids, for foods and dietary supplements may enhance our understanding of the associations between the total antioxidant intake and human health, as suggested by a few studies ${ }^{(43,68)}$. Furthermore, the estimated intake of dietary antioxidants does not always reflect the bioavailability of these antioxidants. Thus, the use of biochemical indicators, for instance, the plasma total antioxidant capacity that considers the antioxidant capacity in the human body, could enhance the efficacy of flavonoid research ${ }^{(69,70)}$.

In conclusion, dietary flavonoid intakes of Korean adults were relatively low compared with other countries and their major food sources were fruits, vegetables and legumes and legume products. The findings of this study could facilitate further investigation on the health benefits of flavonoids among Koreans and provide the basic information for establishing recommended flavonoid intakes for Koreans.

\section{Acknowledgements}

This work was supported by the National Research Foundation of Korea (NRF) grant funded by the Korea government (MSIP) 
(no. 2014R1A2A2A01003138). NRF had no role in the design, analysis or writing of this article.

The authors' responsibilities were as follows: H. J. formulated the research questions and critically reviewed the manuscript; S. S. contributed to the research design; S. J. carried out database construction, data analysis and wrote the draft of the manuscript.

There are no conflicts of interest.

\section{References}

1. Kumar S \& Pandey AK (2013) Chemistry and biological activities of flavonoids: an overview. ScientificWorldJournal 2013, 162750.

2. Kumar S, Sharma UK, Sharma AK, et al. (2012) Protective efficacy of Solanum xanthocarpum root extracts against free radical damage: phytochemical analysis and antioxidant effect. Cell Mol Biol 58, 174-181.

3. Kumar S, Mishra A \& Pandey AK (2013) Antioxidant mediated protective effect of Parthenium hysterophorus against oxidative damage using in vitro models. BMC Complement Altern Med 13, 120

4. Beecher GR (2003) Overview of dietary flavonoids: nomenclature, occurrence and intake. J Nutr 133, 3248S-3254S.

5. Manach C, Williamson G, Morand C, et al. (2005) Bioavailability and bioefficacy of polyphenols in humans. I. Review of 97 bioavailability studies. Am J Clin Nutr $\mathbf{8 1}$ 230S-242S

6. Williamson G \& Manach C (2005) Bioavailability and bioefficacy of polyphenols in humans. II. Review of 93 intervention studies. Am J Clin Nutr 81, 243S-255S.

7. McCullough ML, Peterson JJ, Patel R, et al. (2012) Flavonoid intake and cardiovascular disease mortality in a prospective cohort of US adults. Am J Clin Nutr 95, 454-464.

8. Hooper L, Kroon PA, Rimm EB, et al. (2008) Flavonoids, flavonoid-rich foods, and cardiovascular risk: a meta-analysis of randomized controlled trials. Am J Clin Nutr 88, 38-50.

9. Wang X, Ouyang YY, Liu J, et al. (2014) Flavonoid intake and risk of CVD: a systematic review and meta-analysis of prospective cohort studies. Br J Nutr 111, 1-11.

10. Zamora-Ros R, Forouhi NG, Sharp SJ, et al. (2013) The association between dietary flavonoid and lignan intakes and incident type 2 diabetes in European populations: the EPIC-InterAct study. Diabetes Care 36, 3961-3970.

11. Wedick NM, Pan A, Cassidy A, et al. (2012) Dietary flavonoid intakes and risk of type 2 diabetes in US men and women. Am J Clin Nutr 95, 925-933.

12. Beking K \& Vieira A (2010) Flavonoid intake and disabilityadjusted life years due to Alzheimer's and related dementias: a population-based study involving twenty-three developed countries. Public Health Nutr 13, 1403-1409.

13. Gao X, Cassidy A, Schwarzschild MA, et al. (2012) Habitual intake of dietary flavonoids and risk of Parkinson disease. Neurology 78, 1138-1145.

14. Romagnolo DF \& Selmin OI (2012) Flavonoids and cancer prevention: a review of the evidence. J Nutr Gerontol Geriatr 31, 206-238.

15. Vermeulen E, Zamora-Ros R, Duell EJ, et al. (2013) Dietary flavonoid intake and esophageal cancer risk in the European Prospective Investigation into Cancer and nutrition cohort. $\mathrm{Am}$ J Epidemiol 178, 570-581.

16. Cutler GJ, Nettleton JA, Ross JA, et al. (2008) Dietary flavonoid intake and risk of cancer in postmenopausal women: the Iowa Women's Health Study. Int J Cancer 123, 664-671.
17. Jacques PF, Cassidy A, Rogers G, et al. (2013) Higher dietary flavonol intake is associated with lower incidence of type 2 diabetes. J Nutr 143, 1474-1480.

18. Nettleton JA, Harnack LJ, Scrafford CG, et al. (2006) Dietary flavonoids and flavonoid-rich foods are not associated with risk of type 2 diabetes in postmenopausal women. J Nutr 136, 3039-3045.

19. Morimoto Y, Maskarinec G, Park SY, et al. (2014) Dietary isoflavone intake is not statistically significantly associated with breast cancer risk in the Multiethnic Cohort. Br J Nutr 112, 976-983.

20. Woo HD \& Kim J (2013) Dietary flavonoid intake and risk of stomach and colorectal cancer. World J Gastroenterol 19, 1011-1019.

21. Hui C, Qi X, Qianyong Z, et al. (2013) Flavonoids, flavonoid subclasses and breast cancer risk: a meta-analysis of epidemiologic studies. PLOS ONE 8, e 54318.

22. Wang L, Lee IM, Zhang SM, et al. (2009) Dietary intake of selected flavonols, flavones, and flavonoid-rich foods and risk of cancer in middle-aged and older women. Am J Clin Nutr 89, 905-912

23. Scholz S \& Williamson G (2007) Interactions affecting the bioavailability of dietary polyphenols in vivo. Int J Vitam Nutr Res 77, 224-235.

24. Williamson G \& Holst B (2008) Dietary reference intake (DRI) value for dietary polyphenols: are we heading in the right direction? Br J Nutr 99, Suppl. 3, S55-S58.

25. Gaine PC, Balentine DA, Erdman JW Jr, et al. (2013) Are dietary bioactives ready for recommended intakes? Adv Nutr 4, 539-541.

26. Erdman JW Jr, Balentine D, Arab L, et al. (2007) Flavonoids and heart health: proceedings of the ILSI North America Flavonoids Workshop, May 31-June 1, 2005, Washington, DC. J Nutr 137, 718S-737S

27. Haytowitz DB, Bhagwat S \& Holden JM (2013) Sources of variability in the flavonoid content of foods. Procedia Food Sci 2, 46-51.

28. Bhagwat S, Haytowitz DB \& Holden JM (2013) USDA database for the flavonoid content of selected foods, Release 3.1. Beltsville, MD: US Department of Agriculture, Agricultural Research Service.

29. Bhagwat S, Haytowitz DB \& Holden JM (2008) USDA database for the isoflavone content of selected foods, Release 2.0, US Department of Agriculture, Agricultural Research Service, Beltsville, MD.

30. US Department of Agriculture (2004) USDA database for the proanthocyanidin content of selected foods, US Department of Agriculture, Agricultural Research Service, Beltsville, MD.

31. Neveu V, Perez-Jimenez J, Vos F, et al. (2010) PhenolExplorer: an online comprehensive database on polyphenol contents in foods. Database (Oxford) 2010, bap024.

32. Rothwell JA, Urpi-Sarda M, Boto-Ordonez M, et al. (2012) Phenol-Explorer 2.0: a major update of the Phenol-Explorer database integrating data on polyphenol metabolism and pharmacokinetics in humans and experimental animals. Database (Oxford) 2012, bas031.

33. Rothwell JA, Perez-Jimenez J, Neveu V, et al. (2013) PhenolExplorer 3.0: a major update of the Phenol-Explorer database to incorporate data on the effects of food processing on polyphenol content. Database (Oxford) 2013, bat070.

34. Dragsted LO, Strube M \& Leth T (1997) Dietary levels of plant phenols and other non-nutritive components: could they prevent cancer? Eur J Cancer Prev 6, 522-528.

35. Chun OK, Chung SJ \& Song WO (2007) Estimated dietary flavonoid intake and major food sources of US adults. J Nutr 137, 1244-1252. 
36. Bai W, Wang C \& Ren C (2014) Intakes of total and individual flavonoids by US adults. Int J Food Sci Nutr 65, 9-20.

37. Yang YJ, Kim YJ, Yang YK, et al. (2012) Dietary flavan-3-ols intake and metabolic syndrome risk in Korean adults. Nutr Res Pract 6, 68-77.

38. Lee HS, Cho YH, Park J, et al. (2013) Dietary intake of phytonutrients in relation to fruit and vegetable consumption in Korea. J Acad Nutr Diet 113, 1194-1199.

39. Yeon JY, Bae YJ, Kim EY, et al. (2015) Association between flavonoid intake and diabetes risk among the Koreans. Clin Chim Acta 439, 225-230.

40. National Academy of Agricultural Science (2009) Tables of Food Functional Composition, 1st ed. Suwon: National Academy of Agricultural Science.

41. Holden JM, Bhagwat SA, Haytowitz DB, et al. (2005) Development of a database of critically evaluated flavonoids data: application of USDA's data quality evaluation system. $J$ Food Compost Anal 18, 829-844.

42. Jung HJ, Han SN, Song S, et al. (2011) Association between adherence to the Korean Food Guidance System and the risk of metabolic abnormalities in Koreans. Nutr Res Pract 5, 560-568.

43. Chun OK, Floegel A, Chung SJ, et al. (2010) Estimation of antioxidant intakes from diet and supplements in US adults. J Nutr 140, 317-324.

44. Zamora-Ros R, Sacerdote C, Ricceri F, et al. (2014) Flavonoid and lignan intake in relation to bladder cancer risk in the European Prospective Investigation into Cancer and Nutrition (EPIC) study. Br J Cancer 111, 1870-1880.

45. Zamora-Ros R, Knaze V, Lujan-Barroso L, et al. (2013) Differences in dietary intakes, food sources and determinants of total flavonoids between Mediterranean and non-Mediterranean countries participating in the European Prospective Investigation into Cancer and Nutrition (EPIC) study. Br J Nutr 109, 1498-1507.

46. Zamora-Ros R, Andres-Lacueva C, Lamuela-Raventos RM, et al. (2010) Estimation of dietary sources and flavonoid intake in a Spanish adult population (EPIC-Spain). I Am Diet Assoc 110, 390-398.

47. Li G, Zhu Y, Zhang Y, et al. (2013) Estimated daily flavonoid and stilbene intake from fruits, vegetables, and nuts and associations with lipid profiles in Chinese adults. $J$ Acad Nutr Diet 113, 786-794.

48. Lee MJ, Popkin BM \& Kim S (2002) The unique aspects of the nutrition transition in South Korea: the retention of healthful elements in their traditional diet. Public Health Nutr 5, 197-203.

49. Irala-Estevez JD, Groth M, Johansson L, et al. (2000) A systematic review of socio-economic differences in food habits in Europe: consumption of fruit and vegetables. Eur J Clin Nutr 54, 706-714.

50. Hur I, Jang MJ \& Oh K (2011) Food and nutrient intakes according to income in Korean men and women. Osong Public Health Res Perspect 2, 192-197.

51. Hanson KL \& Connor LM (2014) Food insecurity and dietary quality in US adults and children: a systematic review. Am J Clin Nutr 100, 684-692.

52. Dehghan M, Akhtar-Danesh N \& Merchant AT (2011) Factors associated with fruit and vegetable consumption among adults. J Hum Nutr Diet 24, 128-134.
53. Friel S, Newell J \& Kelleher C (2005) Who eats four or more servings of fruit and vegetables per day? Multivariate classification tree analysis of data from the 1998 Survey of Lifestyle, Attitudes and Nutrition in the Republic of Ireland. Public Health Nutr 8, 159-169.

54. Vogiatzoglou A, Mulligan AA, Luben RN, et al. (2014) Assessment of the dietary intake of total flavan-3-ols, monomeric flavan-3-ols, proanthocyanidins and theaflavins in the European Union. Br J Nutr 111, 1463-1473.

55. Knaze V, Zamora-Ros R, Lujan-Barroso L, et al. (2012) Intake estimation of total and individual flavan-3-ols, proanthocyanidins and theaflavins, their food sources and determinants in the European Prospective Investigation into Cancer and Nutrition (EPIC) study. Br J Nutr 108, 1095-1108.

56. Wang Y, Chung SJ, Song WO, et al. (2011) Estimation of daily proanthocyanidin intake and major food sources in the US diet. J Nutr 141, 447-452.

57. Otaki N, Kimira M, Katsumata S, et al. (2009) Distribution and major sources of flavonoid intakes in the middle-aged Japanese women. J Clin Biochem Nutr 44, 231-238.

58. Ovaskainen ML, Torronen R, Koponen JM, et al. (2008) Dietary intake and major food sources of polyphenols in Finnish adults. J Nutr 138, 562-566.

59. Dilis V \& Trichopoulou A (2010) Antioxidant intakes and food sources in Greek adults. J Nutr 140, 1274-1279.

60. Arai Y, Watanabe S, Kimira M, et al. (2000) Dietary intakes of flavonols, flavones and isoflavones by Japanese women and the inverse correlation between quercetin intake and plasma LDL cholesterol concentration. J Nutr 130, 2243-2250.

61. Messina M, Nagata C \& Wu AH (2006) Estimated Asian adult soy protein and isoflavone intakes. Nutr Cancer 55, 1-12.

62. Perez-Jimenez J, Fezeu L, Touvier M, et al. (2011) Dietary intake of 337 polyphenols in French adults. Am J Clin Nutr $\mathbf{9 3}$, $1220-1228$

63. Zhang Y, Li Y, Cao C, et al. (2010) Dietary flavonol and flavone intakes and their major food sources in Chinese adults. Nutr Cancer 62, 1120-1127.

64. Zamora-Ros R, Knaze V, Lujan-Barroso L, et al. (2011) Estimation of the intake of anthocyanidins and their food sources in the European Prospective Investigation into Cancer and Nutrition (EPIC) study. Br J Nutr 106, 1090-1099.

65. Kita J, Tada J, Ito M, et al. (2004) Intake of phytochemicals among Japanese, calculated by the new FFF database. Biofactors 22, 259-263.

66. Zamora-Ros R, Knaze V, Romieu I, et al. (2013) Impact of thearubigins on the estimation of total dietary flavonoids in the European Prospective Investigation into Cancer and Nutrition (EPIC) study. Eur J Clin Nutr 67, 779-782.

67. Pietta PG (2000) Flavonoids as antioxidants. J Nat Prod 63 , 1035-1042.

68. Yang M, Chung SJ, Chung CE, et al. (2011) Estimation of total antioxidant capacity from diet and supplements in US adults. Br J Nutr 106, 254-263.

69. Schlesier K, Harwat M, Bohm V, et al. (2002) Assessment of antioxidant activity by using different in vitro methods. Free Radic Res 36, 177-187.

70. Wang Y, Chun OK \& Song WO (2013) Plasma and dietary antioxidant status as cardiovascular disease risk factors: a review of human studies. Nutrients 5, 2969-3004. 